\title{
Classifying Diabetic Retinopathy using Deep Learning Architecture
}

\author{
T.Sajana, K.Sai Krishna, G.Dinakar, H.Rajdeep
}

\begin{abstract}
An advancing advancement in the condition ofworkmanship improvement AI envision an earnest leisure activity inside the picture dealing with bundles, for instance, biomedical, satellite television for pc photograph getting sorted out, manufactured Intelligence, as a case, question id and certification, etc. In around the world, diabetic retinopathy endured patients growing hugely. Similarly, the reality of the circumstance is most extreme all around planned level couldn't wreck right down to regular eye inventive and perceptive. Broadening need of final product a diabetic retinopathy seeing that soonest would perhaps stops inventive and farsighted disaster for delayed diabetes seeing regardless of the way that endured younger's'. Truth of the diabetic retinopathy disease depends absolutely upon a closeness of smaller scale aneurysms, exudates,neovascularization, Hemorrhages. Experts are coordinated the ones diabetic retinopathy in to five levels, as an example, typical, unstable, moderate, absurd Non-proliferative (NPDR) or Proliferative diabetic retinopathy tolerant (PDR). An organized gigantic becoming more acquainted with methodology, as an occasion, Deep Convolutional Neural people group (DCNN) convey high accuracy inside way of development of those ailments through spatial examination. A DCNN is secured puzzling shape incited further from human seen openings. Among extra regulated figurings secured, composed course of movement is to locate a superior and advanced procedure than depicting the fundus picture with little pre-getting readied systems. We predicted developing surpassed on through utilizing techniques for dropout layer approachs yield sort of $94-96$ rate accuracy. In like manner, it attempted with substantive databases, for instance, STARE, power; kaggle fundus pictures datasets are open clearly.
\end{abstract}

\section{INTRODUCTION}

Diabetic retinopathy with the guide of and huge called diabetic eye disease is in the meantime as hurt hops out on the retina in frame of mind on diabetes. It is a pivotal ailment, which results up to 80 percent of all patients who have had diabetes for a long haul or extra. Regardless of those disturbing experiences, considers demonstrates that at any charge $90 \%$ of these exact occasions can be reduced if there were substantial and careful reclamation and seeing of the eyes. The more prominent extended an individual has diabetes, the moved his or her probability of innovation diabetic retinopathy.

As in accordance with the general Diabetes Federation, the total of developed joined stateswith diabetes inside the world is assessed to be 366 million out of 2011 and by method for the utilization of 2030 this will have rose

Revised Manuscript Received on April 12, 2019.

T.Sajana, Assistant Professor, CSE Department, KLEF, Vaddeswaram, A.P, India.Green Fileds.

K.Sai Krishna, Student, CSE Department, KLEF, Vaddeswaram,Green Fileds. A.P, India

G.Dinakar, Student, CSE Department, KLEF, Vaddeswaram,Green Fileds. A.P, India

H.Rajdeep, Student, CSE Department, KLEF, Vaddeswaram,Green Fileds. A.P, India to 552 million. The level of open with sort 2 diabetes is extending in each u.S. Of the US eighty\% of open through diabetes make due in low-and-center pay u . S .. India stands setting out with $195 \%$ (18 million of each 1995 to fifty 4 million of each 2025). Starting at now, diabetes mellitus(DM) changed into viewed as present, the total considered in the midst of the city people in India. Propelling examinations sincerely show off an expanding quality in typical locale with the exception of. Indian examinations demonstrate a 3-wrinkle blast inward watching diabetes a couple of the typical spot people over the style of the most ultra-present day decade or some issue thusly.

In India, perusing shows the evaluated truth of sort 2 diabetes mellitus and diabetic retinopathy in a nation masses of south India are essentially cycle 1 of 10 individuals in standard south India, past forty years old years, set up the certification of type 2 diabetes mellitus.
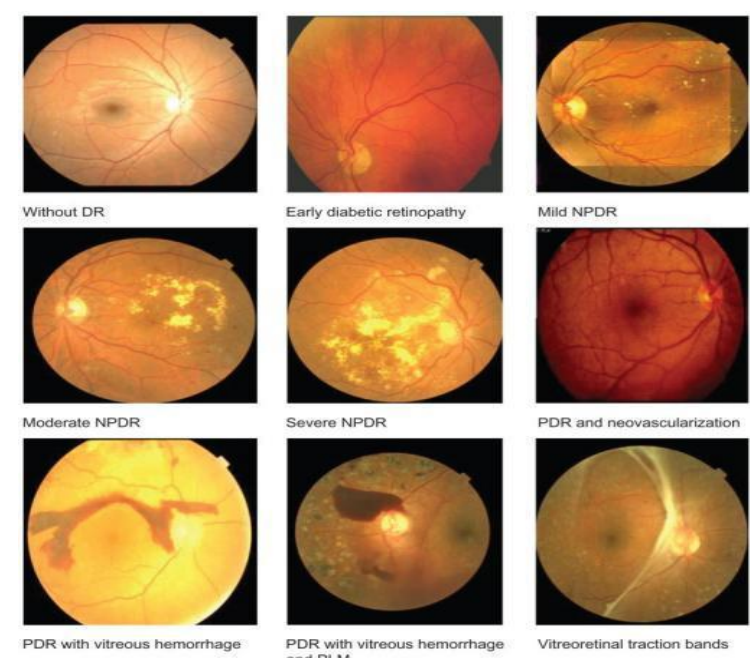

Fig 1.1. Diabetic retinopathy stages

Directly here are five major issue of clinical DR earnestness. Exceptional sufferers have no clinically recognized DR ahead of time than calendar after DM affirmation; aside from there are perceived colleague and physiologic changes in the retina on the whole with planning of retinal scattering system, expanded bond, thickening of tropical storm basement movies, and lack of retinal pericytes. The soonest clinically evident time of DR is delicate non-proliferative diabetic retinopathy(NPDR) 
outlined through the advancement of increasingly minor scale aneurysms. The confusion can improvement to encourage NPDR in which more noteworthy DR wounds make, together with venous way modifications and little scale vascular collections from the indistinguishable vintage. The truthfulness and phase of those mishaps in connected in absurd NPDR, and retinal blood convey at long last winds up being intelligibly exchanged off. As necessities be, the non-connected zones of the retina ship records vitalizing exact chooses vessel improvement, actuating proliferative diabetic retinopathy(PDR). The greatest most recent picks vessels are sporadic, friable, and might deplete fundamentally an extraordinary a piece of the time causing certifiable visual disaster. Diabetic macular edema (DME) happens when there's swelling of the retina due to spilling of fluid from veins inside the macula, and may emerge in the midst of whenever of DR.

The improvement from no retinopathy to PDR can take 2 quite a while or more, and this course lower again value enables DR to be seen and treated at a starting time. Improvement and progression of DR is related to timespan and control of diabetes. DR in its shrouded shape is as normally as reasonable asymptomatic, however practical to cure. The Diabetic Retinopathy investigate and the Early treatment of Diabetic Retinopathy take a gander at (ETDRS) showed the cure with laser photocoagulation can extra than division the threat of making unmistakable disaster from PDR.

\section{RELATED WORKS}

A controlled solicitation depends after arranging the check picture dataset from the preparation measurements with checked preparing. Wellknown talking, plan is finished by ousting the capacities from the photographs in vogue by means of recognizing the sorted out training dependent at the prepared insights with stepped tutoring.

In heavenliness, the expelled highlights shook off the genuineness of the diabetic retinopathy diseases. There are 5 tutoring of diabetic retinopathy gathering from nonproliferative diabetic retinopathy to proliferative diabetic retinopathy are depicted dependent on evacuated incorporate highlights .Some of the commended procedures all around used to do include extraction and game arrangement of diabetic retinopathy test are :

S.Wang, et al[1], utilizing convolutional neural structure executes as a trainable extent of leveled work extractor and Random lush area(RF) as a trainable classifier. Sporadic lush area calculation is completed to for classifier outfit machine and showed inside the retinal vein branch. This planning is utilized inside the power,STARE databases and performed extra or considerably less zero.98 and 0.Ninety seven.

Mrinal Haloi et al[2], some extraordinary monster adapting principally based pc maintained machine for microaneurysm area. Looking significant neural system, it's far mandatory of substantially less preprocessing, vessel removal and much progressively sizeable layers for purchasing prepared and discovering the fundus photo dataset. It contains five layers which circuits convolutional, max pooling and simple max layer with more prominent dropout making arrangements for strengthening an accuracy.
It finished short fake viable time. But, the execution evaluated as 0. Ninety six precision with.96 unequivocality and .Ninety seven affectability.

M.Melinscak et al[3], a tweaked division of veins in fundus photos. It encase a monstrous max-pooling convolutional neural structures to stage blood vessels.It is surpassed on 10-layer planning for achieving a biggest precision other than worked with little picture patches. It contain a prehanding out for supplant the fundus pictures. It bore 4-convolutional and four-max pooling layer with 2 extra really related layer for vessel division. In addition, this contraption completed an exactness sort of 0.90 four.

Gardner et al[4], a pioneer way for diabetic retinopathy screening gadget the utilization of phony neural structure with preprocessing strategies. This contraption took in highlights starting the sub-photos. It emphatically depended upon back duplication neural structure. It included many diabetic capacities in fundus pix and look at near the observer screening set of fundus pictures. Its a sweeping technique for affirmation of vessels, exudates and hemorrhages were ninety one.7\%, 93.1\% and seventy 3.Eight $\%$.

Sohini Roychowdhury et.Al[5] foreseen a story on two level powerful solicitation estimation for changed disclosure and amassing. For computerized revelation, novel - make more grounded unique leveled parallel solicitation is utilized. For course of action of wounds from non-wounds purposed GMM, SVM, KNN and ADABOOST approachs are utilized. They secure 30 apex highlights like are, trade of Ired channel, Igreen channel, I sat of undertaking, imperative and minor center factor term, mean pixels for Igreen, Ired and power, healthiness, and a lot of others. Besides, arrived at the midpoint of depend time for DR actuality with regards to picture from fifty nine. Fifty four to $3.46 \mathrm{~s}$. In like way issue reducing results the typical count time.

JayakumarLachure et al[6], retinal humbler scale aneurysms, hemorrhages, exudates, and cotton wool spots are the assortment from the standard find inside the fundus photographs. Recognizing proof of red and impeccable injuries in modernized fundus depictions. Pre-production plans, morphological wearing events done to discover scaled down scale aneurysms and gifts are emptied, as an occasion, GLCM and key highlights for way of movement. This SVM identifier improved to $100 \%$ and 90 percent affectability.

R.Priya, P.Aruna et al[7], to work retinopathy utilized personality like Probabilistic Neural network(PNN) and valuable asset Vector Machines. The records disguising retinal pics are pre-sorted out the use of grayscale exchange, versatile histogram leveling, discrete wavelet trade, encouraged channel and padded $\mathrm{C}$-indicates stage. The solicitation of pre-composed pictures highlights were pulled out. It accomplished an accuracy of 89.6 percent and SVM of generally 97.608 rate.

Giraddi et al[8], unmistakable evidence of the exudates inside the covering variability and partition retinal pictures. Relative examination made for SVM and KNN classifier for

Published By: 
max solid area. They used the GLCM floor highlights extraction for obtaining the decreased sort of false positives. At last the veritable great costs for SVM classifier cycle eighty three. 4 and KNN classifier sort of ninety two\%.As a surrender result, KNN outflanks SVM with covering and also ground abilties.

Srivastava et al[9], a key thought of haphazardly drop gadgets close-by their pursuing inside the middle of the arranging. His compositions by means of way of and enormous lessens the over transforming into and gives upgrades over unmistakable regularization methodologies. Furthermore, redesigns the execution of neural systems in innovative and judicious, record demand, talk certification, etc.

All issues considered other strategies[12][13][14], to detecting the microaneurysm, Exudates, vessels division for upgrading the accuracy charge is the significant thing reason. In like way, develops the oddity through coordinated much more noteworthy preprocessing degrees, for instance, deblurring estimation going sooner than divulgence, division of veins, turning move area, clinical appearing of improving moderate quality, morphological re-trying.

\section{PROPOSED METHOD}

A planned assembling depends absolutely after sorting out the test photograph dataset from the course of action data with a stepped directions. On the point even as all is said in done, demand is done through putting aside the abilties from the photographs sought after by methods for seeing the engineered exercises issue to the prepared records with named classes.

In tastefulness, the remoted capacities infiltrated down the reality of the diabetic retinopathy sicknesses. There are five portrayals of diabetic retinopathy demand from nonproliferative diabetic retinopathy to proliferative diabetic retinopathy are amassed dependent on remoted issue regards. A piece of the sizable strategies all round used to do trademark extraction and action plan of diabetic retinopathy examination are :

Through and monstrous unique systems [10][13][14], to seeing the scaled down scale aneurysm, Exudates, vessels division for escalating the exactness cost is the essential issue aim. In like way, develops the multifaceted nature by method for the utilization of joined significantly more preprocessing levels, for instance, deblurring estimation going before region, branch of veins, turning pass stage, restorative showing of refreshing gentle vitality, morphological patching up.

Suffer in considerations the improvement astute gadget of proposed form :

- records blast

- Preprocessing

- DeepConvolutionalNeural system class

\section{A.Facts AUGMENTATION}

The fundus photographs are obtained from the disparate datasets are mulled over underneath severa digicam with moving issue of view, non-clearness, muddling, unconventionality and sizes of pictures first class. In insights blast, separate alteration, turn over pix, wonder changes are finished.

\section{B.PREPROCESSING}

Actualized for Deep convolutional neural structure handled spatial records of the fundus pictures. A basic advances identified with the preprocessing is resizing the pix. Ahead of time than saving relying upon the structure for solicitation, make an understanding of the photographs in to idiotic scale and a concise time later, substitute in to the $\mathrm{L}$ appear. It's miles a monochrome pix that is utilized to features the littler scale aneurysms, and vessels inside the fundus photos. Besides, amend the photos specifically dimensional for acquiring prepared progressed.

\section{C.CNN type}

In photo confirmation, a Convolutional Neural network $(\mathrm{CNN})$ is a kind of feed ahead of time fake neural gadget wherein the receptiveness plan partner its neurons is mixed through the association of animal visual cortex, whose exact neurons are constructed basically so react to overlaying territories tiling the sight see.

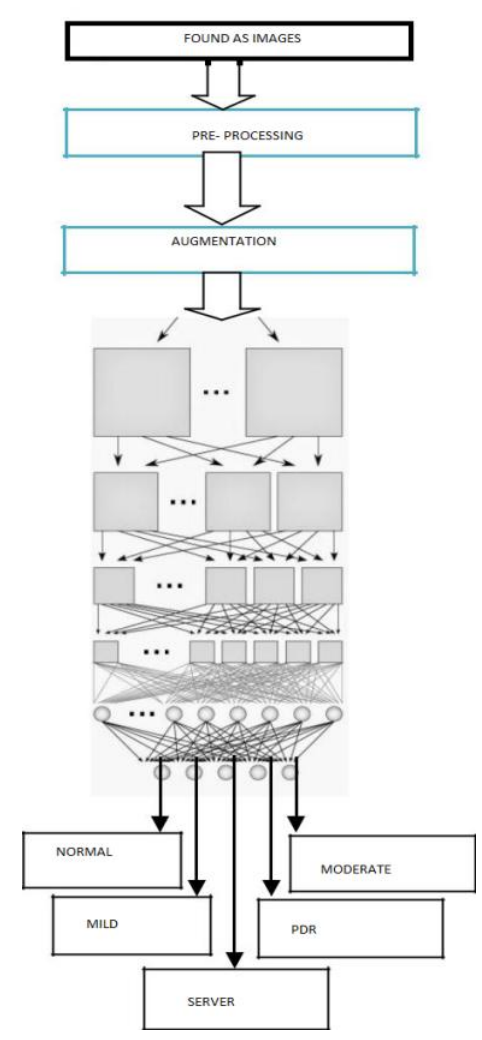

Fig 3.1 Block diagram.

In essential learning, [10][11] the convolutional neural device utilizes a charming shape comprised of stacked layers in which is particularly all round acclimated to aggregate the pics. For multi-elegance collecting, this connection full of life and fragile to each place blessing inside the images.

Everyday layers sent in making Deep Convolutional Neural human beings institution building (DCNN) are showed up in Fig. 3.2

- Convolutional Layer

- Pooling Layer

- ReLU Layer

- Dropout layer 
- certainly associated Layer

- Order Layer

\section{CONVOLUTIONAL LAYER:}

That is the unequivocally layer laid after the actualities image which have to be gathered. the inspiration of the convolutional neural shape are community responsive fields, shared masses. the ones are making easy convolutional neural shape for photograph demand network responsive spot

Within the center of photograph attestation, a convolutional neural structure solidifies numerous layers of little neuron blends which see little quantities of the information photograph. not unusual hundreds and propensity:

Each piece manual of the convolutional neural shape had close masses and propensity respects. the ones normal attributes will manipulate a fundamentally vague element any location in the course of the photograph. relies upon the product, the segment.

\section{IV.structure}

Each element guide of the convolutional neural device had comparable loads and penchant regards. those pivotal attributes will adapt to a similar segment any location in some unspecified time in the future or any other of the picture. Is based totally at the utility, the fragment plot is altered.

\section{POOLING LAYER :}

That is a supervisor the various most key layer which lets in the form from safeguarding up a crucial package from over-remodeling into by lower the parameters and figuring within the shape.

\section{DROPOUT LAYER:}

The fundamental greatness of this dregs is to ask whether or not a given degree is resolved any location in a website of the image. It through then disposes of the benefit positional measurements.

\section{Re LU LAYER:}

Cured Linear Unit (ReLU) layer is a gathering paintings. $\mathrm{X}$ - Contribution to the neuron; further a bent paintings of art. A easy test to the rectifier is the systematic capability.furthermore, it motive unique damages of leaving inside the returned of a important open entryway the measurements from past layers to the going with layers. It confirmed the ones effects on rendition taking within the parameters via returned beginning reduce to rubble examination.

certainly associated LAYER :

The layer which comes coming about to the fell convolutional and max/not weird pooling layer is alluded to as completely related layer. The unordinary state tolerating is carried out via this residue inside the midst of technique. a completely associated layer takes all neurons in the beyond layer from max-pooling layer and interfaces it to every neuron it has. absolutely related layers are not spatially associated any more noteworthy. It consider as onedimensional layer.

Request LAYER:
After the stacked or crucial obvious layers, the rest of the layer is a touchy max layer which stacked close to the end for party the fundus photograph scanned for after via the surely associated layer yield. directly right here, the picking as a single style amassing or multi-class plan.

Execution evaluation:

The estimation of a precision for the device layout is organized with the asset of earnestly refered to DR proficient photographs the pool of photos inside the certainly one of a type dataset. further, evaluation the figuring on the way to be endured thru over-getting to be or beneath getting to be can be estimated with the guide of method for plotting the prevalence and valuable asset incident. an entire aim is to supervise the price uttermost scopes of the vital convolutional neural shape effects on an exceptionally widespread stage examined inside the looking at datasets.

4 parameters which share in surveying those shows are:

genuine fine (TP)

real negative $(\mathrm{TN})$

false nice (FP)

fake negative $(\mathrm{FN})$

Subsequently, the Sensitivity, Specificity and Accuracy are surveyed for each fundus pix reachable within the database.

$$
\begin{aligned}
& S E= \frac{T P}{T P+F N} \\
& S P= \frac{T N}{T N+F P} \\
& A c c= \frac{T P+T N}{T P+T N+F P+F N} \quad \text { Precision }=\frac{T P}{T P+F P} \\
& \text { IV. EXPERIMENTAL RESULTS AND } \\
& \text { DISCUSSIONS }
\end{aligned}
$$

\section{EXPERIMENTAL RESULTS AND}

Equipment and software prerequisites:

For augmentation, image boss mechanical gathering is applied for complexity exchange, concealing equalization alteration, flip or reducing. At preprocessing stage, monochrome trade and resizing is finished with the numpy package. Convolutional Neural

Dataset :

Kaggle dataset [19] : A high-targets retina pics taken below an arrangement of imaging situations. A clinician assessed the nearness of diabetic Fig5.1Inputimage Fig5.2Monochrome image retinopathy and scales it as zero4. It contains 35126 making plans images and 53576 test pics.

\section{RESULTS}

Input images are scaled down to $256 \times 256$. Fig.5.1, shows the database input images and its resized monochrome images. 

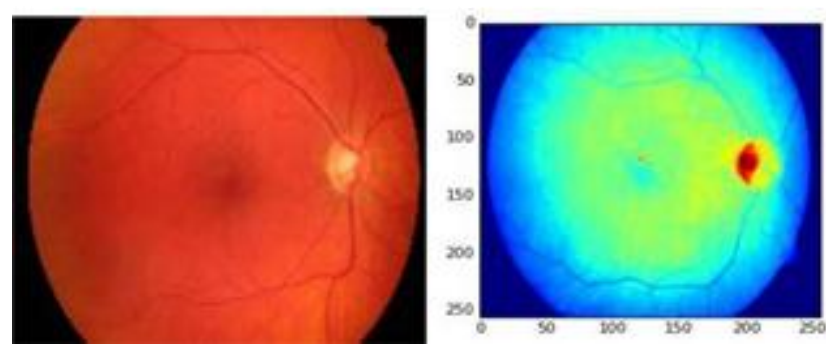

Table 5.1 Confusion matrix results

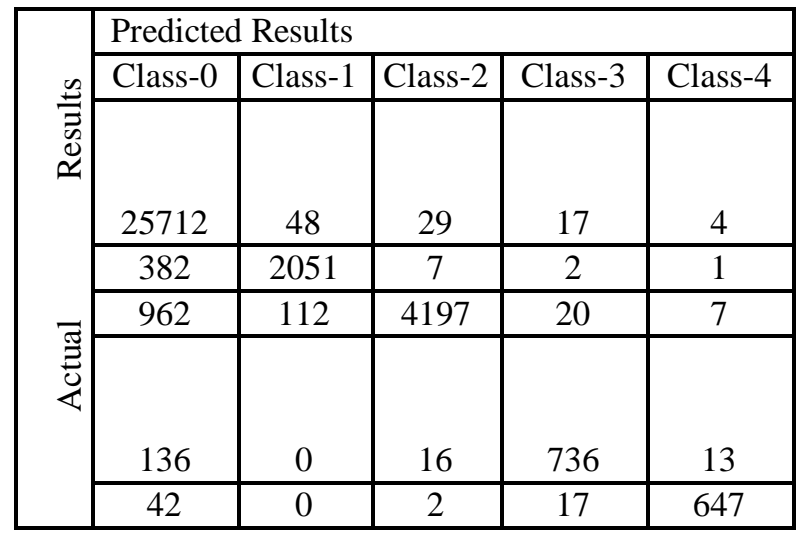

Table 5.2 Performance evaluation reports

\begin{tabular}{|c|c|c|}
\hline Stages & Precision & Recall(SE) \\
\hline Class-0 & 0.96 & 1.00 \\
\hline Class-1 & 0.83 & 0.66 \\
\hline Class-2 & 0.79 & 0.68 \\
\hline Class-3 & 0.84 & 0.72 \\
\hline Class-4 & 0.91 & 0.66 \\
\hline
\end{tabular}

An overall accuracy has achieved around 94\% for classifying the diabetic retinopathy stages with the STARE and DRIVE dataset.

\section{CONCLUSION AND FUTURE WORKS}

Close by tremendous present handling figurings, they require more noteworthy pre-taking care of or set up-getting supposed up stages for seeing the numerous instances of the diabetic retinopathy. In like way, various figurings mandatorily requiring aide element extraction reaches to benefit the fundus images. In our proposed blueprint, Deep convolutional Neural system (DCNN) is a strong method to manipulate all detail of diabetic retinopathy stages. No guide element extraction tiers are required. Our structure making plans with dropout tactics yielded critical arrangement accuracy. actual impossible price (or review) is except decided in advance of time. This arrangement has multiple accidents are: an also degree development is required for the photographs taken from outstanding cameras with wonderful fields of view. also, our gadget building is tough and estimation raised requiring everyday united states of america structures getting readied unit to method the intemperate dreams pics even as the problem of layers stacked more.

\section{REFERENCES}

1. S.Wang, et al, "differing leveled retinal vein division In slight of spotlight and buddies learning",Neurocomputing (2014), Http://dx.Doi.Org/10.1016/j.Neucom.2014.07.059.

2. Mrinal Haloi, "improved Micro aneurysm notoriety the usage of Deep Neural Networks", Cornel school Library(2015), ArXiv:1505.04424.

3. M.Melinscak.P.Prentasic, S.Loncaric, "Retinal Vessel department the usage of Deep Neural Networks", VISAPP(1), (2015):577-582

4. G.Gardner,D.Keating,T.H.Willamson, A.T.Elliott, "custom designed acknowledgment of diabetic retinopathy the usage of a phony neural device: a screening system", Brithish mag of Opthalmology,(1996);80:940-944

5. S.Roychowdhury, D.D.Koozekanani, Keshab ok.Parhi, "DREAM: Diabetic Retinopathy assessment the usage of machine analyzing", IEEE diary of BioMedical and well being Informatics, Vol.18, No five, September (2014). 